\title{
A Revised Trade-to-trade Model for all levels of Trading Thinness in Event Studies
}

\begin{abstract}
This paper offers an improvement to the trade-to-trade model for event studies. While the trade-totrade model of Maynes and Rumsey (1993) addresses the problem of thin trading by eliminating periods in which no trading is recorded, the proposed improvement addresses the influence of zero-value returns resulting from liquidity trading. This entails segmentation by the sign of company returns (positive, negative, zero). The approach allows for all levels of thinness in security trading. It is evaluated against the trade-to-trade methodology developed by Maynes and Rumsey (1993) and the Market Model using Monte Carlo simulations developed from the method of Brown and Warner (1980 and 1985). The improved trade-to-trade model is better at picking up the presence of very small levels of abnormal performance.
\end{abstract}

JEL Classification: C32, G14.

Key words: Event study methodology, trade-to-trade model, Monte Carlo simulations, thin trading 


\section{Introduction}

Thin trading is found on most of the world's stock exchanges. Indeed, if an exchange is not one of the world's biggest, then the zero-value returns associated with either thin trading or mere liquidity trading may well be one of its most obvious characteristics. In the case of the Australian and Thai stock exchanges for instance, Corrado and Truong (2008) documented the incidence of zero value returns at close to 46 and 44 percent respectively. In the current study, we find the incidence on New Zealand data to be 54 percent. A preponderance of zero-value returns causes event study statistical output to be distorted; and the history of searches for an answer to this problem forms one strand of the modern development of event study methodology. This paper contributes to that literature by presenting an improved trade-to-trade model for generating abnormal returns for use in event studies on thinly traded stocks that also exhibit zero-value returns from liquidity trading.

Zero-value returns can occur in two ways. The first is where a zero-value return is used to denote a trading period in which no trade occurs. This is handled in existing trade-to-trade methodology by shortening the data set by excluding such days from the record. The second type of zero-value return results from the failure of the price to shift, from close to close, even by one up- or down-tick in a day's trading, which is evidence of liquidity trading only. This kind of zero-value return is left in place by the trade-to-trade model, which therefore potentially deals with only half of the problem. The model proposed in this paper addresses this problem by segregating company and market return pairs by the sign of the company return to minimise the impact of zero-value returns generated by liquidity trading. The paper demonstrates that this makes the trade-to-trade methodology more accurate in detecting abnormal performance if and when it exists in an event study's event window.

Enhancements like this are of value because event studies are a much-used research tool. The number of event study papers is not known, but five major finance journals between them accounted for 565 
instances between 1974 and 2000 (Kothari and Warner, 2007, p.7). ${ }^{1}$ It is likely the number would be even greater if phenomena affecting stocks at the thinner end of the trading spectrum could be processed with a more thin-friendly methodology. In particular, this would be a boon for research in smaller financial markets. Further, any improvements in accuracy could also be considered to make economic sense, since event studies are now being used as a forensic tool employed by the legal profession (Bhagat and Romano 2007). This alone would demand that the methodology be as refined and trustworthy as possible.

\section{Review of the Literature}

Worries about thinness of trading and the deleterious effect it could have on regression results via biased beta estimates were first raised by Fisher (1966). Initially this was seen as an issue concerning the use of monthly data in the calculation of the cost of equity, where bias occurs as a result of the nonsynchronicity, inside the month, of final company and market returns for the month. Among the first to suggest solutions were Scholes and Williams (1977) and Dimson (1979). But even this early, the focus shifted to the biasing of betas caused by a stock's possible failure to trade at all in a period, or worse still, over multiple consecutive periods. This kind of failure is problematic for event study researchers, as the biasing of betas distorts the magnitude of the abnormal returns used in measuring the impact of an event study's event.

The problem of missing trades became more bothersome with increasing use of daily data in the 1980s; and although they were not specifically designed for it, Scholes and Williams' and Dimson's models tended to be seen as the panaceas for addressing it. However, Brown and Warner (1985) found neither could outperform the conventional market model (a simple ordinary least squares regression) in the

\footnotetext{
${ }^{1}$ The journals Kothari and Warner surveyed were the Journal of Finance, Journal of Business, Journal of Financial Economics, Journal of Financial and Quantitative Analysis, and Review of Financial Studies. They note (p. 6) that the actual number of event studies published will be much greater than this.
} 
accurate detection of abnormal performance. ${ }^{2}$ Fowler, Rorke, and Jog (1989) went on to demonstrate that neither model eliminated the biasing of betas. Cowan and Sergeant (1996) then provided corroboration of Brown and Warner's findings, reinforced by Bartholdy and Riding (1994), who showed that the market model produced betas that were as consistent as those of Scholes and Williams and of Dimson, while superior in efficiency and smallness of bias.

However, another common way of dealing with thin trading is to use the simple market model while excluding from the study any company that trades too infrequently. The downside of exclusion is that it limits the available sample and could make it less representative Zmijewski (1984). Also implicit in this approach is the assumption that the problem of missing trades in non-excluded companies has been finessed in some way at the data level, either by the researcher, or by the data provider. With respect to data sets from the United States, an appendix to Lesmond, Ogden and Trzinka (1999) provides an excellent insight into the quite complex manner in which CRSP decides what to put in the gaps left by missing trades. Elsewhere in the world, time series of closing prices often have these gaps left in them, or data providers (for instance, DataStream) simply bridge the gaps with repetitions of the most recent actual closing price to where the next one occurs. This parlays the missing trades into zero-value returns in the time series. Maynes and Rumsey (1993) describe this as the "lumped" method, and both they and Kallunki (1997) assess it and another hole-filling methodology called the "uniform" method in preparation for OLS processing. ${ }^{3}$ But the lumped approach in particular is likely to load large numbers of zero-value returns into data sets with the likely effect that the variance of returns will be understated, which will cause tests of the abnormal returns to yield biased statistics (Bartholdy, Olson, and Peare 2007).

\footnotetext{
${ }^{2}$ Brown and Warner (1985) simply remove non-trading days and the day that trade resumes entirely from analysis. Thus it was not an ideal method and was superseded by the advent of the trade-to-trade model with rank test proposed by Maynes and Rumsey (1993)

${ }^{3}$ The uniform method entails calculating the multiperiod return from the last trade to the next, dividing by the number of non-trading days in the span, and apportioning the dividend to each.
} 
If zero value returns from non-trading, then, are seen as the problem, there are two ways of dealing with them. One is to get rid of them by dropping non-traded periods, which is what the trade-to-trade model does. The trade-to-trade model was initially developed in the 1970s and documented by Marsh $(1979)^{4}$ and Dimson and Marsh (1983). It became important when Maynes and Rumsey (1993) paired it up with an improvement on the rank test developed by Corrado (1989). ${ }^{5}$

The work of Maynes and Rumsey (1993) and the various papers by Corrado need to be seen in the context of another development in the research record, in which they have been major participants. This is the Brown-and-Warner study as a research tool. This determines the accuracy not just of models, but of the significance test methodologies that render their results meaningful.

It was first used by Brown and Warner (1980) and involves running Monte Carlo simulations on actual, but randomly selected stock market price data, where each simulation is in the format of an event study's set of calculations on an entirely artificial 'event'. The event is manufactured by the researcher, who induces abnormal performance by adding a known percentage increase to the company return for the period designated as the event window. Maynes and Rumsey, and Corrado involved themselves in the debate started by Brown and Warner as to what is the optimal model and test combination in event studies generally. In the original paper in the genre, Brown and Warner (1980) used simulations employing 250 fifty-firm portfolios to evaluate the relative abilities of mean adjusted returns, market adjusted returns and the market model to detect various levels of abnormal performance when paired up with a $t$-test or the Wilcoxon signed rank test. Brown and Warner (1985) reconsidered the same

\footnotetext{
${ }^{4}$ Marsh noted previous uses of the trade-to-trade method by Schwert (1977) and Franks, Broyles, and Hecht (1977).

${ }^{5} \mathrm{~A}$ search in June 2010 that was restricted to papers written in English, uncovered 59 citations of Maynes and Rumsey (1993).
} 
three items in simulations on daily data, and found them to be almost as accurate as the market model. ${ }^{6}$ They also examined the effect of an increase in variance in the event window and found the null hypothesis was rejected too often when no actual abnormal performance was present.

A partial, but not perfect solution for the increased variance problem came to hand when Boehmer, Musumeci, and Poulsen (1991) proposed a standardized cross-sectional $t$-test that took account of increased variance of abnormal returns in the test period. ${ }^{7}$ They found it to be more reliable, in simulations with 250 fifty-firm portfolios, than any other known parametric method in the presence of event-induced increase in variance tested. Corrado (1989) achieved the same end with a new rank test (with the market model), which he demonstrated on 1,200 portfolios of 10, 30 and 50 stocks. Then, in quick succession, Corrado and Zivney (1992) proved that Corrado's rank test out-performed an improved version of the sign test on sets of 1,200 fifty-firm and 10-firm portfolios; and Maynes and Rumsey (1993) adapted Corrado's rank test to be compatible with the trade-to-trade model. With 395 forty-stock portfolios, this too was presented as a Brown-and-Warner-style study.

Further 1990s studies in the Brown-and-Warner style include Cowan (1992), Campbell and Wasley (1993), and Kallunki (1997) whose investigation of the lumped method was mentioned earlier. An important contribution was also made by Cowan and Sergeant (1996). These authors used NYSE-AMEX and NASDAQ data from CRSP to compare two parametric tests and two non-parametric tests in conjunction with the market model and Scholes and Williams' (1977) nonsynchronous trading adjustment model. The parametrics were a $t$-test with a prediction error adjustment proposed by Patell (1976) and Boehmer et al's (1991) standardized cross-sectional $t$-test; and the nonparametrics were

\footnotetext{
${ }^{6}$ A mean adjusted return is the days' company return minus its average $\left(R_{j t}-\bar{R}_{j}\right)$ market adjusted return is simply the day's company return minus the market return (i.e., $R_{j t}-R_{M t}$ ). As shown by Brown and Warner (1980 and 1985) the increase in predictive power of the Market Model over these other models is small. With respect to the mean adjusted returns model, this implies that share prices oscillate about the stock's perceived 'true' value.

${ }^{7}$ This test was also independently developed by Sanders and Robins (1991).
} 
Corrado's (1989) rank test and Cowan's (1992) generalized sign test. Cowan and Sergeant found that the rank and sign tests had greater power and were better specified than the two parametric tests for identifying abnormal performance on all trading thicknesses of stocks. However, when they loaded an increase in variance onto their event day abnormal returns, they found that none of the tests performed well. However, with respect to thinness of trading, Cowan and Sergeant's assessment of their tests' powers was somewhat indirect. Given that they were using time series data already 'corrected' for nontrading by CRSP, they used trading volume as a proxy for thinness, while rejecting all series that had more than 12 missing values.

More recently, Bartholdy, Olson, and Peare (2007) perform a similar comprehensive analysis, but with a much more direct approach to examining thin trading effects. And they employ the trade-to-trade model. They assess it against the market model, where both are partnered up with eight candidate test methodologies, and find it the better model. Three of the test methodologies are permutations of $t$ tests dating from Brown and Warner (1980 and 1985), which turn out to be of little account. The fourth and fifth are the standardized rank test and sign test from Corrado and Zivney (1992). The sixth is the generalized sign test from Cowan (1992) examined by Cowan and Sergeant (1996). The final two are Boehmer et al's (1991) standardized cross-sectional $t$-test and the rank test used by Maynes and Rumsey (1993). To select winners in this eight-way contest, Bartholdy et al run simulations on 1,000 portfolios of 10, 25 and 50 stocks listed on the Copenhagen Stock Exchange sorted into three bands of trading thinness, with zero, $1 / 2$ percent and 2 percent abnormal performance added. With zero abnormal performance added for thickly-traded firms (80 percent or more of the time), they find Boehmer et al's standardized cross-sectional $t$-test to be the most accurate, slightly more so than Maynes and Rumsey's rank test. When abnormal performance is added, Boehmer et al's test yields consistent results but is dominated by all of the nonparametric tests. The two rank tests dominate the other six candidates especially when portfolio size is reduced to 25 and then 10 stocks. However, with the imposition of both 
a $1 / 2$ percent abnormal performance and a 50 percent increase in variance, none of the tests do particularly well. Indeed, with increased variance, two of the three Brown and Warner t-tests tests perform least badly (which is still poorly), and the authors conclude that one should not trust test results unless the level of abnormal performance that the test 'validates' is 2 percent or greater.

Corrado and Truong (2008) performed a Brown-and-Warner study of the performance of the market model in conjunction with a battery of five significance tests on ten Asian exchanges, the Australian Stock Exchange and the three US exchanges (NYSE, AMEX and NASDAQ). The battery comprised two parametric tests (Patell (1976) and Boehmer et al (1991)), a bootstrap test, and Corrado and Zivney's (1992) rank and sign tests. In addition they evaluated the relative merits of arithmetic versus logarithmic returns, and equal-weighted versus value-weighted market indices. The best overall combination Corrado and Truong found was when the market model was run with an equal-weighted index and linked with the rank test. They noted, however, that the use of logarithmic returns did mitigate model misspecification induced by the use of a value-weighted index. However, Corrado and Truong did not tackle the problem of thin trading head-on. They restricted their sample to series that would yield estimation periods (p. 409) "satisfying $200-N_{M}-N_{Z} \geq 150$, where $N_{M}$ is the number of missing returns and $\mathrm{N}_{\mathrm{Z}}$ is the number of zero returns in the 200-day trading period." This set the maximum allowance for thin trading in the sample at 25 percent only.

A comprehensive review of the methodologies mentioned so far is provided in Corrado (2009). ${ }^{8}$

The second major way of dealing with the zero-value returns, whether associated with non-trading or liquidity trading, is to segregate them. This idea is used in friction modeling adapted for use in finance research. Friction models, proposed first by Rosett (1959), were developed for use in various fields of economics (Dagenais 1969; Dagenais 1975; Desarbo et al. 1987; Forbes and Mayne 1989; Almekinders

\footnotetext{
${ }^{8}$ Corrado (2009, pp8-10) mentions more than 25 Brown and Warner-style papers, excluding those of Brown and Warner (1980 and 1985).
} 
and Eijffinger 1996; Hashimoto and Takatoshi 2004). Noting that such a model could be applied to share trading behavior, Lesmond, Ogden, and Trzcinka (1999) built one to determine round-trip transactions costs on stock trading. The salient feature of their friction model is that stock returns remain at or close to zero over a bounded range while being significantly different from zero once those bounds are exceeded. Anderson (2009) used their friction model to replace the market model in an event study employing data from a relatively thinly-traded market. However, the friction model entails maximum likelihood estimation and use of numerical optimisation procedures which are not always user-friendly.

Nevertheless, the concept of working with returns sorted into three regions (positive, negative and zero) can be imported into OLS regression. This idea of partitioning was used by Jokung and Meyfredi (2003) and Norsworthy, Gorener, Morgan and Li (2004). Both studies sorted returns into four categories (by sign of both company and market returns) in a family of 4-state models; but these did not entail any specific categorization of zero-value returns.

The specific categorization of zero value returns is the point that distinguishes the current paper's proposed model from that of Maynes and Rumsey (1993) and its predecessors. The trade-to-trade model drops trading periods in which no trading happens and stitches those that remain back together. The proposed model goes one step further by distinguishing between the effects of positive and negative company returns and that of zero-value returns resulting from liquidity trading. Hence, let's call it the signed trade-to-trade model. To demonstrate its value, we compare its performance with two other models: Maynes and Rumsey's (1993) trade-to-trade model and the market model with missing trades handled by the lumped method. Further, in the manner of Bartholdy, Olson, and Peare (2007), we run our comparison on three categories of trading frequency, employing a range of significance tests. The trading frequency categories are thickly traded (trading on 80 percent of trading days), medium (between 40 and 79 percent of trading days) and thinly traded (less than 40 percent of the 
time). The tests are Boehmer et al's standardized cross-sectional $t$-test, Corrado and Zivney's (1992) rank test and Cowan (1992) sign test.

The rest of the paper is laid out as follows: In Section 2 the methodology is explained, while Section 3 covers the results. Section 4 deals with conclusions and limitations.

\section{Data and Methodology}

We will start with the models, then move on to the significance tests, and from there to a description of the data sets used in this study and the methodology of the Brown-and-Warner-style simulations. As there are rather a lot of variables, an initial glossary may be useful:

Table 1: Variables

\begin{tabular}{|c|c|}
\hline$A R_{j 0}$ & $\begin{array}{l}\text { Abnormal return for company } j \text { on the event day which is actually } t_{144} \text { of the } 250 \text {-day } \\
\text { time series when numbered } t_{1} \text { to } t_{250}\end{array}$ \\
\hline$\overline{\overline{A R_{0}}}$ & Mean of abnormal returns on the day of the event (day zero) \\
\hline$A R_{j t}^{\text {Hadj }}$ & $\begin{array}{l}\text { Heteroscedasticity-adjusted abnormal return for company } j \text { at time } t \text { used in the trade- } \\
\text { to-trade models }\end{array}$ \\
\hline$A R_{j}^{M E D}$ & Median abnormal return for company $j$ for both estimation and test periods $\left(t_{1}\right.$ to $\left.t_{250}\right)$ \\
\hline$E V_{j}^{+}, E V_{j}^{-}$ & Values assigned by the positive and negative operators in Equation 19 \\
\hline$M_{j}$ & The number of returns included in the rank test for company $j\left(t_{1}\right.$ to $\left.t_{250}\right)$ \\
\hline$N$ & Number of companies $(j)$ in the sample \\
\hline$N_{t}$ & Number of non-missing returns at time $t$ \\
\hline $\operatorname{Prop}_{j}$ & The proportion of positive abnormal return observations for the entire period, $t_{1}$ to $t_{250}$. \\
\hline$Q_{1}, Q_{2}$ & Dummy variables used in the signed trade-to-trade model \\
\hline $\bar{R}_{j}, \bar{R}_{M}$ & $\begin{array}{l}\text { Mean of observations of the return on company } j \text { or on the market included in the } \\
\text { estimation period where inclusions are some subset of }\left(t_{1} \text { to } t_{239}\right)\end{array}$ \\
\hline$S()$ & $\begin{array}{l}\text { Standard deviation of (bracketed variable). The Supercripts pertain to specific } \\
\text { formulations that are provided }\end{array}$ \\
\hline$\left(s^{+}\right),\left(s^{-}\right)$ & $\begin{array}{l}\text { Operators for the positive and negative cases in the assigning of the given values on the } \\
\text { RHS of Equations } 16 \text { and } 17 \text {, with respect to the sign test. }\end{array}$ \\
\hline$S R_{j 0}$ & Security j's standardised residual on the event day by Boehmer et al's method \\
\hline$T^{\text {CAR }}$ & Number of days covered by a cumulative abnormal return \\
\hline$T^{E \& T}$ & Total number of days (250) over the estimation and test periods whether traded or not \\
\hline$T_{j}$ & Number of observations of returns on company $j$ in the estimation period $\left(t_{1}\right.$ to $\left.t_{239}\right)$ \\
\hline $\bar{U} U_{j t}$ & Rank statistic for company $j$ at time $t$ \\
\hline
\end{tabular}




\subsection{Models}

\subsubsection{Market model}

The market model is the traditional workhorse for computing a measure of reaction by investors to a given news event occurring in known event window, which may be one day $\left(t_{0}\right)$, or more in size. The expected return, $\mathrm{E}\left(R_{t}\right)$ is calculated from the parameters estimated in the OLS regression, where the two inputs, daily company return $\left(R_{t}\right)$ and return on the market $\left(R_{M t}\right)$ are natural logs:

$$
\begin{aligned}
& R_{t}=\alpha+\beta R_{M t}+\varepsilon_{t} \\
& E\left(R_{t}\right)=\hat{\alpha}+\hat{\beta} R_{M t}
\end{aligned}
$$

Equation 2

Abnormal returns are then forecasted for the test period containing the event window:

$$
A R_{t}=R_{t}-E\left(R_{t}\right)
$$

Equation 3

As mentioned earlier, missing trades are handled by the lumped method. Since data providers may have already "fixed" the missing trade problem, we start by cleaning the data of these pre-existing fixes. This entailed defining as missing prices all adjusted closing prices that are associated with zero trading volume. Missing prices are replaced by the value of the most recent adjusted closing price resulting from an actual trade, which, when converted into log returns, yield a zero-value return for each nontraded day the market is open.

\subsubsection{Trade-to-trade model}

The existing trade-to-trade model's strength is that it can handle data sets with missing trades - which it does by ditching them (and associated market index observations) and sewing the existing trading observations together as a shorter time-series. Thus it employs multi-period returns where the periods 
are of different length and the number of observations is likely to be different for every company in the sample. The returns, $R_{t}$ and $R_{M t}$ are calculated:

$$
\begin{aligned}
& R_{t}=\ln \left(\frac{P_{t}}{P_{t-n}}\right) \\
& R_{M t}=\ln \left(\frac{\text { Index }_{t}}{\text { Index }_{t-n}}\right)
\end{aligned}
$$

Equation 5

The value of $n$ is the total number of trading days since the stock last actually traded, and $S$ is a measure of each of these untraded days. It is necessary for the trade-to-trade model to adjust for the heteroscedasticity that these untraded days induce in the regression error terms, which is done by multiplying both the dependent and independent variables by $\frac{1}{\sqrt{n}}$ and the intercept term by $\sqrt{n}$ :

$$
\frac{1}{\sqrt{n}} R_{t}=(\sqrt{n}) \alpha+\beta \frac{1}{\sqrt{n}} R_{M t}+\frac{1}{\sqrt{n}} \sum_{s=0}^{t-1} \varepsilon_{s}
$$

Equation 6

From this, the heteroscedasticity-adjusted expected return is:

$$
E\left(\frac{1}{\sqrt{n}} R_{t}\right)=\left(\sqrt{n} \hat{\alpha}+\frac{1}{\sqrt{n}} \hat{\beta} R_{M t}\right)
$$

This furnishes an abnormal return in the form:

$$
A R_{t}^{H A d j}=\frac{1}{\sqrt{n}} R_{t}-\left(\sqrt{n} \hat{\alpha}+\frac{1}{\sqrt{n}} \hat{\beta} R_{M t}\right)
$$




\subsubsection{Signed trade-to-trade model}

This builds on the trade-to-trade model by assigning the company and market return pairs by the sign of each company return into three categories: positive, negative, and zero-value. This sorting entails the employment in the model of two dummy variables: $Q_{1}$, which takes on the value ' 1 ' when $R_{t}$ is positive, and zero otherwise, and $Q_{2}$, which takes on the value ' 1 ' only when $R_{t}$ is negative. $\beta_{Q 1} Q_{1}$ and $\beta_{Q_{2}} Q_{2}$ are both intercept terms. The model for regression becomes:

$\frac{1}{\sqrt{n}} R_{j t}=(\sqrt{n}) \beta_{Q 1} Q_{1}+(\sqrt{n}) \beta_{Q 2} Q_{2}+\frac{1}{\sqrt{n}} \beta_{Q 1 R_{M t}} Q_{1} R_{M t}+\frac{1}{\sqrt{n}} \beta_{Q 2 R_{M t}} Q_{2} R_{M t}+\frac{1}{\sqrt{n}} \sum_{s=0}^{t-1} \varepsilon_{s}$

Equation 9

Here the return expectation adjusted for heteroscedasticity is:

$E\left(\frac{1}{\sqrt{n}} R_{j t}\right)=(\sqrt{n}) \beta_{Q 1} Q_{1}+(\sqrt{n}) \beta_{Q 2} Q_{2}+\frac{1}{\sqrt{n}} \beta_{Q 1 R_{M t}} Q_{1} R_{M t}+\frac{1}{\sqrt{n}} \beta_{Q 2 R_{M t}} Q_{2} R_{M t}$

Equation 10

From this the heteroscedasticity-adjusted abnormal return is calculated:

$$
A R_{j t}^{\text {Hadj }}=\frac{1}{\sqrt{n}} R_{j t}-E\left(\frac{1}{\sqrt{n}} R_{j t}\right)
$$

Equation 11

The zero state is dropped out of the dummy regression procedure on the ground that a zero company return is deemed to be both its own expected return and abnormal return. The slopes of the positive and negative company returns are independent of each other. Both an absence of trading and trading without any price changes convey similar information. This is that investors have not observed any event to warrant trading in the firms' shares at a different price. Thus a zero return can never generate a significant abnormal return. This is a bit of bald-faced normative thinking; but can one really claim that a positive abnormal return exists on an event day if the stock fails to trade and the market return on the 
day happens to be negative? Similarly, when a zero return occurs as a result of trading with an unchanged price, this, in efficient markets parlance, means no new information on this firm has entered the market - or at least no news that is noteworthy to traders. Therefore if investors have not reacted, there cannot be a meaningful abnormal return.

\subsection{Tests of significance}

\subsubsection{Standardized cross-sectional t-test $\left(T_{1}\right)$}

This test was developed by Boehmer, Musumeci, and Poulsen (1991) with a view to mitigating the effect of increased variance in the event window, building on the adjustment procedure of Patell (1976). Patell's adjustment is in the second argument of the denominator of $S R_{j 0}$ below:

$$
T_{1}=\frac{\frac{1}{N} \sum_{j=1}^{N} S R_{j 0}}{\sqrt{\frac{1}{N(N-1)} \sum_{j=1}^{N}\left(S R_{j 0}-\sum_{j=1}^{N} \frac{S R_{j 0}}{N}\right)}}
$$

Equation 12

Where:

$$
S R_{j 0}=\frac{A R_{0}}{\left(\sqrt{\left.\frac{\sum_{t=1}^{T_{j}}\left(A R_{j t}-\bar{R}_{j}\right)^{2}}{N-1}\right)\left(\sqrt{1+\frac{1}{T_{j}}+\frac{\left(R_{M 0}-\bar{R}_{M}\right)^{2}}{\sum_{t=1}^{T_{j}}\left(R_{M t}-\bar{R}_{M}\right)^{2}}}\right)}\right.}
$$

Because this is the only parametric test in the paper, the cross-sectional standardized cross-sectional $t$ test will simply be called the $t$-test. Further, it should be noted that cross-sectional standardization is also impounded into the two nonparametric tests. 


\subsubsection{Sign test $\left(T_{2}\right)$}

This test is specified according to its formulation by Corrado and Truong (2008) with the negative case and a maximum function added in the current paper.

$T_{2}=\max \left[\left(\frac{\left(\sum_{j=1}^{N} \frac{\left(1 \mid A R_{j, 0}>0\right)-\text { Prop }_{j}^{+}}{\sqrt{\left(\text { Prop }_{j}^{+}\right)\left(1-\text { Prop }_{j}^{+}\right)}}\right)}{(\sqrt{N})}\right),\left(\frac{\left(\sum_{j=1}^{N} \frac{\left(1 \mid A R_{j, 0}<0\right)-\text { Prop }_{j}^{-}}{\sqrt{\left(\text { Prop }_{j}^{-}\right)\left(1-\text { Prop }_{j}^{-}\right)}}\right)}{(\sqrt{N})}\right)\right]$

Equation 14

Where:

$$
\left\{\begin{array}{l}
\operatorname{Prop}_{j}^{+}=\frac{\left(\sum_{t=1}^{T_{j}}\left(s^{+}\right)\left(A R_{j t}-A R_{j}^{M E D}\right)\right)}{M_{j}} \\
\operatorname{Prop}_{j}^{-}=\frac{\left(\sum_{t=1}^{T_{j}}\left(s^{-}\right)\left(A R_{j t}-A R_{j}^{M E D}\right)\right)}{M_{j}}
\end{array}\right.
$$

Equation 15

Where

$$
\begin{aligned}
& \left(s^{+}\right)\left(A R_{j t}-A R_{j}^{M E D}\right)=\left\{\begin{array}{l}
1 \text { if } A R_{j t}>A R_{j}^{M E D} \\
0 \text { otherwise }
\end{array}\right. \\
& \left(s^{-}\right)\left(A R_{j t}-A R_{j}^{M E D}\right)=\left\{\begin{array}{l}
1 \text { if } A R_{j t}<A R_{j}^{M E D} \\
0 \text { otherwise }
\end{array}\right.
\end{aligned}
$$

Equation 16

Equation 17

In extreme thin trading, it is possible to have either no positive abnormal returns or possibly no negative ones. If this occurs, the affected proportion is set to 0.5 , which yields the lowest possible $t$-value.

\subsubsection{Rank test $\left(T_{3}\right)$}

The rank test used here is based on the Wilcoxon rank test and an early development from it by Corrado (1989) improved in Corrado and Zivney (1992), and used in a number of subsequent papers (Maynes and 
Rumsey 1993; Kallunki 1997; Bartholdy et al. 2007; Corrado and Truong 2008; Corrado 2009). We start by defining the rank statistic, $U_{j t}$ :

$$
U_{j t}=\frac{\operatorname{rank}\left(A R_{j t}\right)}{\left(1+M_{j}\right)}
$$

Equation 18

In Equation 18, $M_{j}$ is the number of returns included in the model for company $j$, and rank is a function that returns fractional rankings. The sum of fractional rankings (with or without ties) returns the same sum as an ordinal ranking without ties. Other ranking methods will not give the same sum and thus could be calculated incorrectly where ties exist. The test statistic is furnished by:

$$
T_{3}=\frac{\left(\frac{\sum_{j=1}^{N}\left(U_{j, 0}-0.5\right)}{S(U)}\right)}{\sqrt{N}}
$$

Equation 19

Where:

$$
S(U)=\sqrt{\frac{\sum_{t=1}^{T_{j}^{E \& T}\left(\frac{\sum_{j=1}^{N}\left(U_{j t}-0.5\right)}{\sqrt{N_{t}}}\right)^{2}}}{T^{E \& T}}}
$$

Equation 20

Here $N_{t}$ denotes the number of firms from the sample actually included in the model on day $t$, since the two trade-to-trade models drop non-trading days out for firms. 


\subsection{Data}

Daily adjusted closing prices of ordinary equity have been collected from three exchanges for the 20year span from January 1990 to the end of November or December 2009. The three exchanges are the NYSE, the New Zealand Stock Exchange (NZSX) and Mexico's stock exchange, the Bolsa Mexicana de Valores (BOLSA). The NYSE data is from CRSP, while DataStream furnished data for the other two. For all three exchanges, we have included all available companies, including firms that delisted. We use NYSE data because its characteristics are generally well known and its stocks are the most heavily-traded in the world. Also, the first paper employing Monte Carlo simulations for the evaluation of abnormal returns detection methods (Brown and Warner, 1980) used it. NYSE data thus provides a benchmark for demonstrating the validity of the paper's models, which are then let loose on the more thinly-traded NZSX and even more thinly-traded BOLSA data sets. Data from the NZSX are included as the NZSX is an example of a small regional exchange on which trading is often thin. Mexican data is included because BOLSA company returns are relatively volatile and its stocks even more thinly traded. The characteristics of the three data sets are summarised in Table 2.

Given that CRSP has a complex method for assigning prices in the absence of trades, the NYSE data was stripped of assigned pricing whenever trading volume was zero. Similarly, where DataStream had filled in missing trades with the last available previous closing price in accordance with what Kallunki (1997) calls "the lumped method", this also was stripped back so that missing trades became again, missing trades. On the other hand, when an observation of positive trading volumes is associated with a price set at zero by the data provider, the trading day is deemed unusable and causes any estimation period sequence containing it to be rejected.

Table 2: Descriptive Statistics 


\begin{tabular}{|c|c|c|c|}
\hline Stock Exchange & NYSE & NZSX & BOLSA \\
\hline Start Date & 02-Jan-1990 & 03-Jan-1990 & 02-Jan-1990 \\
\hline End Date & 31-Dec-2009 & 30-Nov-2009 & 31-Dec-2009 \\
\hline No. of Trading Days & 5,043 & 5,004 & 5,043 \\
\hline No. of Securities & 3,193 & 132 & 654 \\
\hline Total number of Returns (excluding missing returns) & $7,753,688$ & 739,548 & $1,044,344$ \\
\hline No. of Non-trading Zero Returns (ie, missing trades) & 54,952 & 204,724 & 564,173 \\
\hline Proportion of Non-trading Zero Returns & $0.71 \%$ & $27.68 \%$ & $54.02 \%$ \\
\hline No. of Trading Zero Returns & 772,910 & 194,115 & 97,167 \\
\hline Proportion of Trading Zero Returns & $9.97 \%$ & $26.25 \%$ & $9.30 \%$ \\
\hline Total No. of Non-zero Returns & $6,925,826$ & 340,709 & 383,004 \\
\hline Proportion of Non-zero Returns & $89.32 \%$ & $46.07 \%$ & $36.67 \%$ \\
\hline Mean No. of Days between Trades & 1.27 & 5.26 & 54.76 \\
\hline Mean of Firms' Log Return St Deviations & 0.03 & 0.03 & 0.03 \\
\hline Mean of Firms' Non zero Log Return St Deviations & 0.04 & 0.08 & 0.09 \\
\hline Mean of Firms' Log Return Skewness & -0.16 & -1.04 & -1.40 \\
\hline Mean of Firms' Log Return Kurtosis & 32.36 & 85.79 & 221.02 \\
\hline \multicolumn{4}{|c|}{$\begin{array}{l}\text { a The total number of returns excludes missing returns. These are defined as observations where the closing price is zero, but the volume of } \\
\text { trading in the stock is above eero. The NZSX and BOSA data sets contained instances of this error. By contrast, a "missing trade" is a trading } \\
\text { day on which a stock is not traded (and volume is zero). }\end{array}$} \\
\hline
\end{tabular}

In Table 2, zero-value returns are divided into whether they are from trades in which the price remained unchanged, or represent missing trades. Depending on which of the paper's models is being used, these missing trades may be assigned a zero value, or alternatively be left out of the model. On the NYSE, missing trades account for less than one percent of the total. The NYSE's incidence of trades with no change in price, at just under 10 percent, is also quite small. By contrast these two percentages in the New Zealand market are much higher and, at almost 28 percent and 27 percent, close to equal. The BOLSA shows missing trades, however, account for 54 percent of its total while unchanged-price trades, at 9 percent, are close to those of the NYSE. 
Also of note in Table 2 is the average time lapse between trades in each market. The $543 / 4$ days for the BOLSA is strongly indicative of a large tail of firms which trade very infrequently. The NZSX also has one, but the $5 \frac{1}{4}$ day average implies it is a much smaller tail. ${ }^{9}$ This makes it a slightly more thinly-traded market than the Toronto Stock Exchange with 3.67 days (Maynes and Rumsey 1993) and the Copenhagen Stock Exchange with 4.17 days (Bartholdy et al. 2007). The $1 \frac{1}{3}$ days for the NYSE shows that there is a tail of thin trading in this market too, but it is miniscule compared with those of the other two markets.

The other item of note in Table 2 is the mean volatility of traded returns. This is approximately three times that of the NYSE ( 3 percent) on both the NZSX and the BOLSA ( 8 percent and 9 percent).

The choice of market index used for each market is based on their accessibility. In the case of the NYSE data, we use the CRSP NYSE Equal-Weighted Market Index (Kyindno: 1000001). For the New Zealand data, it is the NZSX all companies gross index, which is value-weighted. (The NZSX does not provide an equal-weighted index.) With respect to the Mexican data, we employ the IPC (BOLSA) index (MXIPC35), which is value weighted.

\subsection{Simulation methodology}

This paper adopts the Monte Carlo simulation format provided by Brown and Warner (1985). For each of the exchanges, 250 portfolios, each containing a 250-day time series for each of 50 stocks, are generated on a random search-with-replacement basis. Any candidate series containing the zero price error are rejected. Over all, three sets of 12,500 pairs of company-return and market-return time-series

\footnotetext{
${ }^{9}$ Nevertheless, this stands in contrast with Bartholdy and Riding (1994, p.245), who noted of their New Zealand data from 1982 to 1987 that their 110 firms traded on 64.1 percent of trading days, and that there was an average lapse of 2.4 days between the last trade and the end of the monthly time measure they used in their study.
} 
are processed. Expected returns are calculated on the first 239 days, and abnormal returns are forecasted for the artificial event window set at day 244 at the centre of an 11-day test period. Because the company return (and associated market return) time series are selected randomly, there will be, on average, no abnormal performance in the event window, which we will call day zero. Artificial abnormal performance is loaded onto day zero so the candidate models can be tested on their ability to reject the null hypothesis of no abnormal performance or not These levels of artificial abnormal performance are $0 \%$ (for finding the rate spurious AR detection), and in order of decreasing size, $\pm 5 \%, \pm 1 \%, \pm 0.5 \%$ and $\pm 0.1 \%$. The 37,500 observation sets selected for the procedure will, at the same time, be used to rate the relative effectiveness of the three significance test methods. This means that for any given level of abnormal performance, results will be obtained for nine model/significance test combinations. Further, in keeping with Bartholdy, Olson, and Peare (2007), we re-examine these nine combinations in a set of further simulations where a new data set is generated according to the specifications of three bands of trading frequency - thick (or frequent) trading, medium and thin trading. These specifications are given in the next section.

\section{Results}

\subsection{Summary statistics in the presence of no induced abnormal return}

Table 3 contains summary of t-statistics when no abnormal performance is added to the observed

return on day zero. Each row contains a sample of 250 computed $t$-statistics, whose distributional properties are shown in columns 4 to 7.

The figures for skewness in column 6 are close to zero and the kurtosis figures in column 5 are generally close to 3 . This indicates the $t$-stats approach a normal distribution - which is predicted by the central limit theorem. 
Table 3: Summary of t-Statistics for Test and Model Combinations

\begin{tabular}{|c|c|c|c|c|c|c|}
\hline (1) & (2) & (3) & (4) & (5) & (6) & (7) \\
\hline Exchange & Test & Model & $\begin{array}{c}\text { Mean of } \\
\text { Means }\end{array}$ & Mean of STD & $\begin{array}{c}\text { Mean of } \\
\text { Skewness }\end{array}$ & $\begin{array}{l}\text { Mean of } \\
\text { Kurtosis }\end{array}$ \\
\hline \multirow[t]{9}{*}{ NYSE } & $t$-test $\left(T_{1}\right)$ & $\mathrm{MM}$ & -0.0681 & 1.0364 & -0.1299 & 2.816 \\
\hline & & T-to-T & -0.0678 & 1.0341 & -0.1228 & 2.8261 \\
\hline & & SignT-to-T & 0.0473 & 1.0394 & -0.2138 & 2.4016 \\
\hline & Rank Test & $\mathrm{MM}$ & -0.3701 & 1.6259 & 0.1899 & 2.9634 \\
\hline & & T-to-T & -0.3596 & 1.6216 & 0.1904 & 2.9972 \\
\hline & & Sign T-to-T & -0.0681 & 1.2315 & 0.1554 & 3.0654 \\
\hline & Sign Test & MM & 0.5866 & 0.5913 & 0.8997 & 3.3105 \\
\hline & & T-to-T & 0.5543 & 0.5975 & 0.9588 & 3.4401 \\
\hline & & Sign T-to-T & 0.6515 & 0.9257 & 0.6571 & 3.6538 \\
\hline \multirow[t]{9}{*}{ NZSX } & $t$-test $\left(T_{1}\right)$ & $\mathrm{MM}$ & 0.1208 & 0.984 & 0.028 & 3.0958 \\
\hline & & T-to-T & 0.0905 & 0.9806 & 0.0591 & 2.973 \\
\hline & & Sign T-to-T & 0.0728 & 0.9737 & 0.0847 & 2.3076 \\
\hline & Rank Test & $\mathrm{MM}$ & 0.2179 & 1.74 & -0.076 & 2.578 \\
\hline & & T-to-T & -0.0189 & 1.4923 & -0.1828 & 2.8039 \\
\hline & & Sign T-to-T & 0.0366 & 1.0537 & 0.3202 & 3.0758 \\
\hline & Sign Test & $\mathrm{MM}$ & 0.5707 & 0.6206 & 0.7393 & 3.0668 \\
\hline & & T-to-T & -0.5216 & 0.6595 & 0.578 & 3.0497 \\
\hline & & Sign T-to-T & 0.3006 & 0.862 & 0.4105 & 3.7279 \\
\hline \multirow[t]{9}{*}{ BOLSA } & $t$-test $\left(T_{1}\right)$ & $\mathrm{MM}$ & -0.0479 & 1.0153 & 0.0245 & 2.6014 \\
\hline & & T-to-T & -0.0834 & 0.992 & -0.0877 & 2.8022 \\
\hline & & Sign T-to-T & 0.0144 & 0.9651 & -0.0602 & 2.49 \\
\hline & Rank Test & MM & -0.6936 & 1.7033 & 0.2346 & 2.8131 \\
\hline & & T-to-T & -0.3087 & 1.3909 & 0.1618 & 2.9649 \\
\hline & & Sign T-to-T & -0.0978 & 1.0472 & 0.2251 & 3.6182 \\
\hline & Sign Test & MM & 0.5939 & 0.6543 & 0.706 & 2.6007 \\
\hline & & T-to-T & -1.2525 & 0.6643 & 0.5987 & 3.6608 \\
\hline & & Sign T-to-T & -0.1679 & 0.7771 & 0.602 & 4.3422 \\
\hline
\end{tabular}

Table 4 contains the characteristics of the residuals furnished in the three markets by the three models. These are broken into results for the estimation period and results for the test period in which day zero is seated. As the results for the test period are a function of forecasting forward from the estimation period, these periods are both set at 125 days in length. 
Table 4: Characteristics of Residuals and Observed versus Expected Returns

\begin{tabular}{|c|c|c|c|c|c|c|c|c|c|}
\hline Exchange & Period & Model & Mean & STD & Skewness & Kurtosis & $\begin{array}{r}\text { Lilliefors } \\
\text { Test }\end{array}$ & $\begin{array}{r}\text { Jarque-Bera } \\
\text { Test }\end{array}$ & $\begin{array}{r}\text { Pearson } \\
\text { Correlation }\end{array}$ \\
\hline \multirow[t]{6}{*}{ NYSE } & Estimation & $\mathrm{MM}$ & 0 & 0.0227 & 0.0543 & 7.4753 & 0.4391 & 0.2554 & 0.3648 \\
\hline & & $\mathrm{T}$ to $\mathrm{T}$ & 0 & 0.0233 & 0.012 & 9.8449 & 0.2271 & 0.0888 & 0.3668 \\
\hline & & Sign $T$ to $T$ & 0 & 0.0153 & 0.0571 & 11.562 & 0.2839 & 0.0874 & 0.7889 \\
\hline & Test & $\mathrm{MM}$ & 0 & 0.0235 & 0.0902 & 7.726 & 0.4402 & 0.2436 & 0.3609 \\
\hline & & $\mathrm{T}$ to $\mathrm{T}$ & 0.0001 & 0.0212 & 0.0878 & 2.8697 & 0.8979 & 0.8654 & 0.3699 \\
\hline & & Sign $\mathrm{T}$ to $\mathrm{T}$ & -0.0002 & 0.017 & 0.0588 & 11.0348 & 0.2499 & 0.1588 & 0.771 \\
\hline \multirow[t]{6}{*}{ NZSX } & Estimation & MM & 0 & 0.0242 & 0.0867 & 8.5521 & 0.178 & 0.1688 & 0.2059 \\
\hline & & $\mathrm{T}$ to $\mathrm{T}$ & 0 & 0.0295 & 0.0816 & 8.4072 & 0.0944 & 0.0831 & 0.2172 \\
\hline & & Sign $T$ to $T$ & 0 & 0.0155 & 0.1319 & 12.9733 & 0.0264 & 0.0453 & 0.8387 \\
\hline & Test & $\mathrm{MM}$ & -0.0001 & 0.0251 & 0.0652 & 9.01 & 0.1756 & 0.1611 & 0.1669 \\
\hline & & $\mathrm{T}$ to $\mathrm{T}$ & 0.0001 & 0.0205 & 0.049 & 2.8351 & 0.8924 & 0.8709 & 0.2357 \\
\hline & & Sign $T$ to $T$ & -0.0004 & 0.0191 & -0.0192 & 12.4482 & 0.0228 & 0.1191 & 0.8114 \\
\hline \multirow[t]{6}{*}{ BOLSA } & Estimation & $\mathrm{MM}$ & 0 & 0.0261 & 0.152 & 10.1351 & 0.1499 & 0.1399 & 0.07 \\
\hline & & $\mathrm{T}$ to $\mathrm{T}$ & 0 & 0.0327 & 0.0821 & 8.0473 & 0.1013 & 0.0979 & 0.074 \\
\hline & & Sign $T$ to $T$ & 0 & 0.0205 & 0.0963 & 8.8169 & 0.1804 & 0.1176 & 0.7646 \\
\hline & Test & $\mathrm{MM}$ & 0 & 0.0265 & 0.0946 & 10.338 & 0.1626 & 0.1436 & 0.0015 \\
\hline & & $\mathrm{T}$ to $\mathrm{T}$ & 0.0006 & 0.0256 & 0.1303 & 2.8346 & 0.8866 & 0.877 & 0.0003 \\
\hline & & Sign $\mathrm{T}$ to $\mathrm{T}$ & -0.0005 & 0.0243 & -0.0001 & 7.8977 & 0.156 & 0.2226 & 0.7328 \\
\hline
\end{tabular}

This table reports the basic properties residuals from the randomly selected 12,500 data-runs from each of the three stock exhanges (37,500 in total). The estimation period is set at 139 days and the test period is 11 days. In each column a mean for each model is reported (for instance, mean kurtosis). The Lilliefors and Jarque-Bera tests of normality results show the proportion of the 12,500 data sets that furnish non-normal residuals. In the final column, the Pearson correlation coefficient shows the mean correlataion between observed returns and estimated returns over the 12,500 runs.

The departure from zero of the average residual means in the test period is simply because of the forecasting forward of expectations. The randomness of data-set selection has ensured that this departure is pretty small. With respect to the three models and NYSE and NZSX data, the signed tradeto-trade exhibits the lowest level of departure from normality as measure by the Lilliefors and JarqueBera tests. The estimation-period Lilliefors test result for the signed trade-to-trade model on the BOLSA is a minor exception to this. In addition, Table 4 provides Pearson correlations that capture the relationship between observed and expected returns provide a measure of each model's goodness of fit. Of immediate note is that the average correlation associated with the signed trade-to-trade model is far higher than those of the other two models for all three markets. However, Table 4 does not furnish a 
result for spurious rejections of the null hypothesis of there being no statistically significant abnormal performance on day zero. This is left for Table 5.

\subsection{Simulations with an induced constant abnormal return}

The rates of rejection of the null hypothesis are shown in Table 5 . We start with a -5 percent induced level of induced abnormal performance, then diminish to -1 percent, and then move from there to the less commonly reported $-1 / 2$ percent and -0.1 percent levels of abnormal performance before repeating these levels as positive increments. For each level, the 250 portfolios (per stock exchange), each containing 50 stocks, furnish 250 test statistics, on each of which an accept/reject decision is made. Each figure is therefore a count of rejections divided by 250 to turn it into a decimal fraction. In the column where zero artificial abnormal performance has been added, the figures should be interpreted as the rate of spurious rejections of the null hypothesis (Type I error). In all columns where non-zero abnormal performance has been introduced, the figures denote the rate of accurate rejection of the null hypothesis. Therefore the incidence of a Type II error will be this rate subtracted from 1.

Panel A covers the results for the three models with respect to Boehmer et al's standardized crosssectional $t$-test. The signed trade-to-trade model consistently furnishes the lowest Type I error of al three models when no abnormal performance is added. For the NYSE, this is 0.016 , reducing to 0.004 on both the NZSX and BOLSA. However, with non-zero induced abnormal returns, the three models tend to produce fairly similar results. 
Table 5: Incidence of Null Hypothesis Rejections by Significance Test with various Levels of Induced Abnormal Performance

\begin{tabular}{|c|c|c|c|c|c|c|c|c|c|c|}
\hline \multirow[b]{2}{*}{ Exchange } & \multirow[b]{2}{*}{ Model } & \multicolumn{9}{|c|}{ Percentage of abnormal performance added } \\
\hline & & $-5 \%$ & $-1 \%$ & $-0.50 \%$ & $-0.10 \%$ & $\mathbf{0}$ & $0.10 \%$ & $0.50 \%$ & $1 \%$ & $5 \%$ \\
\hline \multicolumn{11}{|c|}{ Panel A: Standardised Cross Sectional t-test } \\
\hline \multirow[t]{3}{*}{ NYSE } & MM & 1 & 0.868 & 0.352 & 0.044 & 0.024 & 0.02 & 0.308 & 0.848 & 1 \\
\hline & T-to-T & 1 & 0.864 & 0.348 & 0.044 & 0.024 & 0.02 & 0.308 & 0.844 & 1 \\
\hline & Sign T-to-T & 1 & 0.64 & 0.204 & 0.024 & 0.016 & 0.012 & 0.24 & 0.672 & 0.996 \\
\hline \multirow[t]{3}{*}{ NZSX } & MM & 1 & 0.792 & 0.264 & 0.016 & 0.02 & 0.036 & 0.308 & 0.812 & 1 \\
\hline & T-to-T & 1 & 0.82 & 0.268 & 0.008 & 0.02 & 0.032 & 0.316 & 0.832 & 1 \\
\hline & Sign T-to-T & 0.988 & 0.836 & 0.504 & 0.016 & 0.004 & 0.028 & 0.552 & 0.868 & 0.992 \\
\hline \multirow[t]{3}{*}{ BOLSA } & $\mathrm{MM}$ & 0.992 & 0.752 & 0.288 & 0.024 & 0.012 & 0.012 & 0.24 & 0.768 & 0.992 \\
\hline & T-to-T & 0.996 & 0.708 & 0.224 & 0.028 & 0.012 & 0.012 & 0.172 & 0.688 & 0.996 \\
\hline & Sign T-to-T & 0.98 & 0.72 & 0.268 & 0.012 & 0.004 & 0.008 & 0.296 & 0.684 & 0.984 \\
\hline \multicolumn{11}{|c|}{ Panel B: Rank Test } \\
\hline \multirow[t]{3}{*}{ NYSE } & MM & 1 & 0.988 & 0.788 & 0.216 & 0.168 & 0.16 & 0.684 & 0.988 & 1 \\
\hline & T-to-T & 1 & 0.988 & 0.78 & 0.224 & 0.168 & 0.16 & 0.68 & 0.988 & 1 \\
\hline & Sign T-to-T & 1 & 0.984 & 0.772 & 0.356 & 0.048 & 0.292 & 0.776 & 0.988 & 1 \\
\hline \multirow[t]{3}{*}{ NZSX } & $\mathrm{MM}$ & 1 & 1 & 0.956 & 0.372 & 0.2 & 0.48 & 0.98 & 1 & 1 \\
\hline & T-to-T & 1 & 1 & 0.952 & 0.54 & 0.12 & 0.592 & 0.976 & 1 & 1 \\
\hline & Sign T-to-T & 1 & 1 & 1 & 1 & 0.02 & 1 & 1 & 1 & 1 \\
\hline \multirow[t]{3}{*}{ BOLSA } & $\mathrm{MM}$ & 1 & 0.996 & 0.98 & 0.54 & 0.224 & 0.292 & 0.972 & 1 & 1 \\
\hline & T-to-T & 1 & 0.992 & 0.984 & 0.836 & 0.08 & 0.624 & 0.968 & 0.996 & 1 \\
\hline & Sign T-to-T & 1 & 1 & 0.992 & 0.988 & 0.02 & 0.976 & 0.996 & 1 & 1 \\
\hline \multicolumn{11}{|c|}{ Panel C: Sign Test } \\
\hline \multirow[t]{3}{*}{ NYSE } & MM & 1 & 0.876 & 0.28 & 0.012 & 0 & 0.016 & 0.208 & 0.816 & 1 \\
\hline & T-to-T & 1 & 0.876 & 0.28 & 0.012 & 0 & 0.016 & 0.2 & 0.824 & 1 \\
\hline & Sign T-to-T & 1 & 0.984 & 0.804 & 0.46 & 0.044 & 0.476 & 0.796 & 0.984 & 0.996 \\
\hline \multirow[t]{3}{*}{ NZSX } & $\mathrm{MM}$ & 1 & 0.968 & 0.652 & 0.06 & 0.004 & 0.076 & 0.74 & 0.98 & 1 \\
\hline & $\mathrm{T}$-to- $\mathrm{T}$ & 1 & 0.976 & 0.784 & 0.2 & 0 & 0.196 & 0.796 & 0.98 & 1 \\
\hline & Sign T-to-T & 1 & 1 & 1 & 1 & 0.024 & 1 & 1 & 1 & 1 \\
\hline \multirow[t]{3}{*}{ BOLSA } & $\mathrm{MM}$ & 1 & 0.964 & 0.792 & 0.116 & 0 & 0.028 & 0.716 & 0.956 & 1 \\
\hline & T-to-T & 1 & 0.96 & 0.796 & 0.368 & 0.024 & 0.324 & 0.84 & 0.96 & 1 \\
\hline & Sign T-to-T & 1 & 1 & 1 & 1 & 0.004 & 1 & 1 & 1 & 1 \\
\hline \multicolumn{11}{|c|}{$\begin{array}{l}\text { In this table each row reports the rate of rejection of the null hypothesis of no abnormal performance present when a specified leve } \\
\text { of artificial abnormal performance has been added. For each market, the results are generated from one set of } 250 \text { portfolios of } 50 \\
\text { stocks employing an estimation period of } 139 \text { days for the market model and a maximum of } 139 \text { days for the trade-to-trade and } \\
\text { signed trade-to-trade models, given their treatment of missing trades. In Panel A, the signficance test is the standardized cross- } \\
\text { sectional t-test. In Panel B, the rank test is used. In Panel C, the significance test employed is the sign test. }\end{array}$} \\
\hline
\end{tabular}

In Panel B with the rank test, we see a marked change. In all but one case, the signed trade-to-trade model produces the lowest rate of Type I and Type II errors. In other words, the model's figures in the no abnormal performance column are uniformly smaller while being almost uniformly larger in all other columns. The exception is with -1 percent added for the NYSE, which is indicative of a one observation 
difference. The model in conjunction with the rank test also accurately detects abnormal performance down to the 0.1 percent level. This is an improvement in accuracy over all models linked with the $t$-test.

In Panel C of Table 5, the significance test is the sign test. Over all three exchanges, the signed trade-totrade model has the lowest level of Type II errors, but not consistently the lowest level of a Type I error. While the sign test appears in this table to yield slightly lower Type II errors than the rank test in Panel B, the rank test is preferred in ensuing sections because its rejection rates tend to be more accurate under the more rigorous conditions we now move to.

\subsection{Simulations on data sets with decreased estimation periods}

In this subsection, the same 250 portfolios of 50 stocks that have been used in all procedures so far, are reprocessed so that returns expectations are based on two much shorter estimation periods. In place of 239 days, estimation periods of 111 days 61 days are trialled. Table 6 shows the incidence of null hypothesis rejections with respect to each category in conjunction with the rank test only. On the NYSE with a 111-day estimation period, the market model outperforms the trade-to-trade model, which in turn outperforms the signed trade-to-trade model in the presence of non-zero artificially induced abnormal performance. This pattern is repeated when the estimation period is shortened to 61 days. In addition, at both shortened lengths of estimation period, the rate of accurate diagnosis of induced artificial performance drops below 50 percent when the artificial performance increment is less than one percent. 
Table 6: Incidence of Null Hypothesis Rejections when the Estimation Period is approximately halved and then quartered

\begin{tabular}{|c|c|c|c|c|c|c|c|c|c|c|c|}
\hline \multirow[b]{2}{*}{ Exchange } & \multirow[b]{2}{*}{ EstPerSize } & \multirow[b]{2}{*}{ Model } & \multicolumn{9}{|c|}{ Percentage of abnormal performance added } \\
\hline & & & $-5 \%$ & $-1 \%$ & $-0.50 \%$ & $-0.10 \%$ & $\mathbf{0}$ & $0.10 \%$ & $0.50 \%$ & $1 \%$ & $5 \%$ \\
\hline \multirow[t]{6}{*}{ NYSE } & 111 & MM & 1 & 0.88 & 0.364 & 0.016 & 0.004 & 0.012 & 0.252 & 0.824 & 1 \\
\hline & 111 & T-to-T & 1 & 0.876 & 0.36 & 0.02 & 0.004 & 0.016 & 0.268 & 0.82 & 1 \\
\hline & 111 & Sign T-to-T & 1 & 0.796 & 0.28 & 0.036 & 0 & 0.028 & 0.304 & 0.78 & 1 \\
\hline & 61 & MM & 1 & 0.808 & 0.244 & 0.012 & 0.004 & 0.004 & 0.172 & 0.78 & 1 \\
\hline & 61 & T-to-T & 1 & 0.808 & 0.228 & 0.012 & 0.004 & 0.004 & 0.172 & 0.788 & 1 \\
\hline & 61 & Sign T-to-T & 0.996 & 0.692 & 0.264 & 0.028 & 0 & 0.024 & 0.18 & 0.668 & 0.992 \\
\hline \multirow[t]{6}{*}{ NZSX } & 111 & MM & 1 & 0.968 & 0.72 & 0.08 & 0.024 & 0.076 & 0.74 & 0.964 & 1 \\
\hline & 111 & T-to-T & 1 & 0.968 & 0.728 & 0.172 & 0.008 & 0.144 & 0.74 & 0.968 & 1 \\
\hline & 111 & Sign $T$-to- $T$ & 1 & 1 & 0.992 & 0.96 & 0 & 0.94 & 0.992 & 1 & 1 \\
\hline & 61 & MM & 1 & 0.968 & 0.568 & 0.036 & 0.004 & 0.032 & 0.52 & 0.94 & 1 \\
\hline & 61 & T-to-T & 1 & 0.964 & 0.636 & 0.124 & 0.004 & 0.088 & 0.568 & 0.912 & 1 \\
\hline & 61 & Sign T-to-T & 1 & 1 & 0.996 & 0.948 & 0 & 0.928 & 0.988 & 1 & 1 \\
\hline \multirow[t]{6}{*}{ BOLSA } & 111 & MM & 1 & 0.956 & 0.688 & 0.104 & 0.02 & 0.024 & 0.62 & 0.956 & 1 \\
\hline & 111 & T-to-T & 1 & 0.952 & 0.692 & 0.3 & 0.008 & 0.172 & 0.608 & 0.92 & 1 \\
\hline & 111 & Sign T-to-T & 1 & 0.992 & 0.932 & 0.76 & 0 & 0.772 & 0.928 & 0.988 & 1 \\
\hline & 61 & MM & 1 & 0.916 & 0.56 & 0.056 & 0.024 & 0.02 & 0.376 & 0.844 & 1 \\
\hline & 61 & T-to-T & 1 & 0.912 & 0.62 & 0.244 & 0.004 & 0.108 & 0.44 & 0.808 & 1 \\
\hline & 61 & Sign T-to-T & 1 & 0.972 & 0.856 & 0.676 & 0 & 0.58 & 0.8 & 0.952 & 1 \\
\hline \multicolumn{12}{|c|}{$\begin{array}{l}\text { In this table the same sets } 250 \text { portfolios of } 50 \text { stocks as in the previous table are reprocessed employing estimation periods of } \\
111 \text { days and } 61 \text { days, denoted by the column heading, EstPerSize. For the market model this implies that } 111 \text { and } 61 \\
\text { observations are employed respectively. For the trade-to-trade and signed trade-to-trade models, these become the } \\
\text { maximum number of available observations, which will, with missing trades be less. The reported figures are the incidence, as } \\
\text { a decimal fraction, of rejections of the null hypothesis of no abnormal performance present given the specified percentages of } \\
\text { artificial abnormal performance added. The signficance test used throughout these results is the rank test. }\end{array}$} \\
\hline
\end{tabular}

However, on the NZSX, the signed trade-to-trade model continues to yield rates of null hypothesis rejection above 90 percent even when the induced abnormal performance is reduced to plus or minus one tenth of one percent. With respect to the NZSX, the trade-to-trade model also outperforms the market model, but its detection rates drop away to the low 70 s percentage range once abnormal performance is set at half of one percent with the estimation period set at 111 days. When the estimation period is halved again to 61 days, the trade-to-trade model's detection rate falters down into the low 60 s and high 50 s range with respect to half a percent of induced abnormal performance.

The BOLSA yields results that are similar to those gained from the NZSX data sets. The signed trade-totrade model outperforms both of the other models, but in this market the detection rate falls below 80 
percent once artificially induced abnormal performance is reduced to plus or minus one tenth of one percent.

The final item of interest in Table 6 is that on the 250 portfolios with shortened estimation periods, the incidence of an incorrect rejection of the null hypothesis was zero for the signed trade-to-trade model for both lengths of estimation period and in all three markets. It is probably only coincidental that this performance is better than that observed with respect to the rank test in Table 5 . However, a 2 percent false rejection rate in Table 5 does equate with only 5 rejections.

\subsection{Simulations employing smaller portfolios}

In this section, the portfolios used so far are discarded and a fresh set is generated with the specification that the number of stocks selected per portfolio be, in the first instance, 30 , which is a 40 percent reduction in the size of portfolios hitherto. A second fresh new set of 250 portfolios is generated containing 20 stocks, and a further set with only 10 stocks. The effect on rejections of the null hypothesis are shown in Table 7.

Over all three exchanges, a reduction of portfolio size from 30 to 10 stocks equates with a reduction of accuracy in the rejection rates when any non-zero level of artificial abnormal performance is added. This reduction is negligible when artificial performance is plus or minus 5 percent but becomes quite severe when the level of artificial performance is reduced to plus or minus half a percent. 
Table 7: Incidence of Rejection of the Null Hypothesis of No Abnormal Performance detected when Portfolios are made smaller

\begin{tabular}{|c|c|c|c|c|c|c|c|c|c|c|c|}
\hline \multirow[b]{2}{*}{ Exchange } & \multirow[b]{2}{*}{ PortSize } & \multirow[b]{2}{*}{ Model } & \multicolumn{9}{|c|}{ Percentage of abnormal performance added } \\
\hline & & & $-5 \%$ & $-1 \%$ & $-0.50 \%$ & $-0.10 \%$ & $\mathbf{0}$ & $0.10 \%$ & $0.50 \%$ & $1 \%$ & $5 \%$ \\
\hline \multirow[t]{9}{*}{ NYSE } & 30 & MM & 1 & 0.936 & 0.64 & 0.196 & 0.16 & 0.168 & 0.476 & 0.904 & 1 \\
\hline & 30 & T-to-T & 1 & 0.94 & 0.644 & 0.192 & 0.16 & 0.156 & 0.472 & 0.904 & 1 \\
\hline & 30 & Sign T-to-T & 1 & 0.888 & 0.568 & 0.232 & 0.072 & 0.176 & 0.516 & 0.872 & 1 \\
\hline & 20 & $\mathrm{MM}$ & 1 & 0.84 & 0.428 & 0.128 & 0.14 & 0.12 & 0.328 & 0.744 & 1 \\
\hline & 20 & T-to-T & 1 & 0.84 & 0.428 & 0.128 & 0.132 & 0.12 & 0.332 & 0.744 & 1 \\
\hline & 20 & Sign T-to-T & 0.988 & 0.732 & 0.416 & 0.164 & 0.06 & 0.116 & 0.36 & 0.696 & 0.992 \\
\hline & 10 & MM & 1 & 0.552 & 0.256 & 0.088 & 0.076 & 0.084 & 0.168 & 0.468 & 1 \\
\hline & 10 & T-to-T & 1 & 0.552 & 0.256 & 0.092 & 0.08 & 0.088 & 0.168 & 0.468 & 1 \\
\hline & 10 & Sign T-to-T & 0.68 & 0.384 & 0.2 & 0.08 & 0.008 & 0.036 & 0.152 & 0.328 & 0.7 \\
\hline \multirow[t]{9}{*}{ NZSX } & 30 & $\mathrm{MM}$ & 1 & 0.988 & 0.848 & 0.312 & 0.188 & 0.408 & 0.896 & 0.996 & 1 \\
\hline & 30 & T-to- $T$ & 1 & 0.968 & 0.84 & 0.388 & 0.104 & 0.456 & 0.876 & 0.984 & 1 \\
\hline & 30 & Sign T-to-T & 1 & 1 & 1 & 0.984 & 0.016 & 0.98 & 1 & 1 & 1 \\
\hline & 20 & MM & 1 & 0.932 & 0.688 & 0.176 & 0.116 & 0.252 & 0.768 & 0.948 & 1 \\
\hline & 20 & T-to-T & 1 & 0.912 & 0.704 & 0.304 & 0.088 & 0.352 & 0.772 & 0.944 & 1 \\
\hline & 20 & Sign T-to-T & 1 & 1 & 0.976 & 0.92 & 0.004 & 0.9 & 0.964 & 0.996 & 1 \\
\hline & 10 & MM & 0.996 & 0.636 & 0.432 & 0.144 & 0.136 & 0.164 & 0.496 & 0.72 & 1 \\
\hline & 10 & T-to-T & 0.992 & 0.652 & 0.436 & 0.164 & 0.068 & 0.196 & 0.48 & 0.692 & 1 \\
\hline & 10 & Sign T-to-T & 0.988 & 0.824 & 0.716 & 0.62 & 0.004 & 0.652 & 0.768 & 0.852 & 0.976 \\
\hline \multirow[t]{9}{*}{ BOLSA } & 30 & $\mathrm{MM}$ & 1 & 0.984 & 0.904 & 0.408 & 0.184 & 0.228 & 0.872 & 0.972 & 1 \\
\hline & 30 & T-to-T & 1 & 0.968 & 0.868 & 0.564 & 0.096 & 0.436 & 0.828 & 0.952 & 1 \\
\hline & 30 & Sign T-to-T & 1 & 0.992 & 0.964 & 0.904 & 0.024 & 0.908 & 0.964 & 0.992 & 1 \\
\hline & 20 & MM & 1 & 0.912 & 0.764 & 0.28 & 0.124 & 0.152 & 0.668 & 0.884 & 1 \\
\hline & 20 & T-to-T & 1 & 0.908 & 0.78 & 0.484 & 0.06 & 0.332 & 0.656 & 0.876 & 1 \\
\hline & 20 & Sign T-to-T & 1 & 0.972 & 0.884 & 0.784 & 0.008 & 0.712 & 0.832 & 0.94 & 1 \\
\hline & 10 & MM & 1 & 0.708 & 0.524 & 0.172 & 0.076 & 0.112 & 0.412 & 0.66 & 1 \\
\hline & 10 & T-to-T & 0.996 & 0.656 & 0.488 & 0.264 & 0.028 & 0.16 & 0.376 & 0.604 & 0.996 \\
\hline & 10 & Sign T-to-T & 0.932 & 0.676 & 0.512 & 0.448 & 0.004 & 0.34 & 0.428 & 0.58 & 0.932 \\
\hline
\end{tabular}

This table furnishes the rate of rejection of the null hypothesis of no abnormal performance present, given the stated levels of artificial abnormal performance added on day zero. Each row's results are furnished by a set of 250 portfolios which have been generated specifically for this table. The size of each portfolio is initially 30 stocks, then 20 stocks, and finally 10 stocks. All sizespecific portfolios are generated independently of each other in the monte carlo simulation process. PortSize denotes the size of each portfolio employed in the generating of results for any given row. The estimation period for return expectations is 139 days for the market model and a maximum of 139 days worth of observations for the trade-to-trade and signed trade-to-trade model. The signficance test used is the rank test.

However, for all three models over all three exchanges, the incidence of null hypothesis rejections also drops with decreasing portfolio size in the presence of zero abnormal performance added.

With respect to the NYSE, the signed trade-to-trade model appears to be the worst performer of the three models at almost every level of non-zero induced abnormal performance; but it also has the smallest rate of false rejections of the null when abnormal performance actually is set at zero. 
On NZSX and BOLSA data, the signed trade-to-trade model continues to furnish the lowest incidence of Type I errors when artificial abnormal performance is zero. However, on these two markets, it also outperforms the trade-to-trade and market models at all levels of induced abnormal performance, when portfolios contain 30 stocks. Indeed on the NZSX and BOLSA, its null hypothesis rejection rates for nonzero abnormal performance never drop below 90 percent. This is also true of the signed trade-to-trade model run on 20-stock portfolios of NZSX data, but not quite so true with respect to the BOLSA. None of the models furnish rejection rates with an acceptably low Type II error when portfolios have only 10 stocks, and the level of induced abnormal performance drops below plus or minus 5 percent. This finding holds for all three markets.

\subsection{Simulations on data sets characterized by thin trading}

A fresh set of portfolios is now generated with the new specification that selection is contingent on trading thinness. Following Bartholdy, Olson, and Peare (2007), we divide each exchange's data set into three bands, generating 250 portfolios of 50 stocks for each band:

1. Thickly-traded where a firm trades on 81 percent or more of the time (201 to 250 days).

2. Medium thinness where a firm trades between 41 and 80 percent of the time (101 to 200 days).

3. Thinly-traded where a firm trades on 40 percent of the time or less ( 100 or fewer days)

As Bartholdy et al note, the thick band equates with trading on at least four days in a five-day trading week, while membership of the thinly-traded band is indicative of trades on two or less days. The NYSE data furnished too few firms outside the thickly-traded band for computation of the lower two bands to be feasible. While these authors look at using a method of trade-to-trade to cover thin trading they still required that there be no missing trades in the test period and a minimum of 10 observed prices in the estimation period. The current paper relaxes this minimum. Where there is at least one but less than 6 observations for a regression, the slope is set to zero and the intercept is set to the mean of those 
values. If there are no observations, the slope and intercept are both set to zero. A missing trade on the event day is handled by a zero return with the artificial abnormal performance added to it.

Table 8: Incidence of Null Hypothesis Rejections using the Rank Test with Bands of Trading Thinness

\begin{tabular}{|c|c|c|c|c|c|c|c|c|c|c|}
\hline \multirow[b]{2}{*}{ Exchange } & \multirow[b]{2}{*}{ Model } & \multicolumn{9}{|c|}{ Percentage of abnormal performance added } \\
\hline & & $-5 \%$ & $-1 \%$ & $-0.50 \%$ & $-0.10 \%$ & $\mathbf{0}$ & $0.10 \%$ & $0.50 \%$ & $1 \%$ & $5 \%$ \\
\hline \multicolumn{11}{|c|}{ Panel A: Rank Test linked with Thick Trading } \\
\hline \multirow[t]{3}{*}{ NYSE } & MM & 1 & 0.908 & 0.448 & 0.052 & 0.02 & 0.016 & 0.236 & 0.836 & 1 \\
\hline & T-to-T & 1 & 0.908 & 0.444 & 0.056 & 0.016 & 0.016 & 0.236 & 0.836 & 1 \\
\hline & Sign T-to-T & 0.996 & 0.848 & 0.324 & 0.032 & 0 & 0.04 & 0.208 & 0.728 & 1 \\
\hline \multirow[t]{3}{*}{ NZSX } & $\mathrm{MM}$ & 1 & 0.956 & 0.644 & 0.08 & 0.04 & 0.064 & 0.636 & 0.952 & 1 \\
\hline & T-to-T & 1 & 0.956 & 0.648 & 0.092 & 0.012 & 0.068 & 0.628 & 0.952 & 1 \\
\hline & Sign T-to-T & 1 & 1 & 0.98 & 0.896 & 0 & 0.888 & 0.988 & 1 & 1 \\
\hline \multirow[t]{3}{*}{ BOLSA } & MM & 1 & 0.896 & 0.472 & 0.06 & 0.02 & 0.004 & 0.388 & 0.828 & 1 \\
\hline & T-to-T & 1 & 0.884 & 0.436 & 0.092 & 0.016 & 0.02 & 0.352 & 0.824 & 1 \\
\hline & Sign T-to-T & 1 & 0.82 & 0.464 & 0.196 & 0 & 0.136 & 0.384 & 0.78 & 1 \\
\hline \multicolumn{11}{|c|}{ Panel B: Rank Test linked with Medium Trading } \\
\hline \multirow[t]{3}{*}{ NZSX } & MM & 1 & 0.996 & 0.968 & 0.14 & 0.02 & 0.304 & 0.988 & 1 & 1 \\
\hline & T-to-T & 1 & 0.996 & 0.924 & 0.588 & 0 & 0.68 & 0.956 & 0.988 & 1 \\
\hline & Sign T-to-T & 1 & 1 & 1 & 1 & 0 & 1 & 1 & 1 & 1 \\
\hline \multirow[t]{3}{*}{ BOLSA } & \begin{tabular}{|l|l}
$\mathrm{MM}$ \\
\end{tabular} & 1 & 1 & 1 & 0.284 & 0.016 & 0.132 & 0.98 & 1 & 1 \\
\hline & T-to-T & 1 & 1 & 0.976 & 0.768 & 0 & 0.664 & 0.948 & 0.992 & 1 \\
\hline & Sign T-to-T & 1 & 1 & 1 & 1 & 0 & 1 & 1 & 1 & 1 \\
\hline \multicolumn{11}{|c|}{ Panel C: Rank Test linked with Thin Trading } \\
\hline \multirow[t]{3}{*}{ NZSX } & $\mathrm{MM}$ & 1 & 1 & 1 & 0.18 & 0.076 & 0.816 & 1 & 1 & 1 \\
\hline & T-to-T & 1 & 1 & 1 & 0.988 & 0 & 0.992 & 1 & 1 & 1 \\
\hline & Sign T-to-T & 1 & 1 & 1 & 1 & 0 & 1 & 1 & 1 & 1 \\
\hline \multirow[t]{3}{*}{ BOLSA } & $\mathrm{MM}$ & 1 & 1 & 1 & 0.776 & 0.032 & 0.448 & 1 & 1 & 1 \\
\hline & T-to-T & 1 & 1 & 1 & 1 & 0 & 1 & 1 & 1 & 1 \\
\hline & Sign T-to-T & 1 & 1 & 1 & 1 & 0 & 1 & 1 & 1 & 1 \\
\hline \multicolumn{11}{|c|}{$\begin{array}{l}\text { The three panels of this table contain the rate of rejection of the null hypothesis of no abnormal performance present when } \\
\text { artificially induced abnormal performance is added when stocks are thickly traded, mediumly traded or thinly traded. The } \\
\text { estimation period for return expectations is } 139 \text { days, which is a maximum achievable by only some thickly traded stocks with } \\
\text { respect to the trade-to-trade and signed trade-to-trade models. Thick trading is defined as trading on } 81 \text { percent or more days, } \\
\text { while thin trading is defined as trading on } 40 \text { days or less. The signficance test used here is the rank test. Porfolios of medium anc } \\
\text { thinly traded NYSE stocks could not be generated without massive re-usage of the few stocks that met the selection criteria. }\end{array}$} \\
\hline
\end{tabular}

We also drop the NYSE with respect to medium and thin trading on the ground that less-than-thickly traded stocks in that market were too few to randomly generate 250 portfolios of 50 stocks without massive duplication. 
Table 8 lays out the performance of the three models linked to the rank test. The signed trade-to-trade model consistently has no Type I error. The incidence of type II errors is only slightly higher than for the other models with respect to the NYSE but is near perfect in the medium and thinly-traded bands (Panels B and C). The accuracy of the signed trade-to-trade model in the context of thinly-traded markets is an important finding. The approach generally taken to dealing with infrequently-traded stocks is to dump them out of the data set. This model offers a viable alternative which, in terms of Zmijewski's (1984) criticism of financial distress detection methodology, would help keep an event study's sample more representative of its underlying population.

\subsection{Simulations on data sets with increased variance in the event window}

Artificial increases in event window variance data back to Brown and Warner $(1985$, p.23) who noted that an increase in event-window variance caused the misspecification of parametric tests used in hypothesis testing. Event-period standard deviations tended to be 1.2 to 1.5 times greater than those of the estimation period (Boehmer et al., 1991, p.257). To investigate the phenomenon in their simulations, Brown ad Warner doubled the variance of the day zero return by adding day -6 into the calculation of a transformed return employed in variance calculation - an approach that was also adopted by Corrado and Zivney (1992), Corrado and Truong (2008) using days +6 and +3 respectively. Boehmer et al used a measure of artificially induced event window variance that entailed scaling the variance of estimation period residuals by a factor of k, which was adopted also by Bartholdy et al. (2007). The current paper follows the latter approach using standard deviations.

In keeping with the range of standard deviation increase cited by Boehmer et al, we initially scale the standard deviation of returns in the event window up by 50 percent. We also return to using the original set of 250 portfolios of 50 stocks used in generating the results furnished from Table 4 to Table 6. 


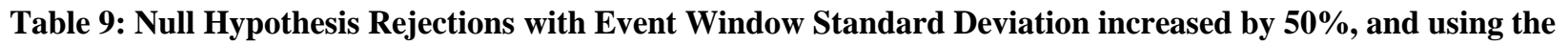
Rank Test

\begin{tabular}{|c|c|c|c|c|c|c|c|c|c|c|}
\hline \multirow[b]{2}{*}{ Exchange } & \multirow[b]{2}{*}{ Model } & \multicolumn{9}{|c|}{ Percentage of abnormal performance added } \\
\hline & & $-5 \%$ & $-1 \%$ & $-0.50 \%$ & $-0.10 \%$ & $\mathbf{0}$ & $0.10 \%$ & $0.50 \%$ & $1 \%$ & $5 \%$ \\
\hline \multicolumn{11}{|c|}{ Panel A: Rank Test, $50 \%$ Increased Variance on Day Zero, and Thick Trading } \\
\hline \multirow[t]{3}{*}{ NYSE } & MM & 1 & 0.744 & 0.224 & 0.06 & 0.028 & 0.008 & 0.132 & 0.624 & 1 \\
\hline & T-to-T & 1 & 0.744 & 0.224 & 0.06 & 0.024 & 0.008 & 0.128 & 0.624 & 1 \\
\hline & Sign T-to-T & 0.996 & 0.476 & 0.12 & 0.004 & 0 & 0.004 & 0.044 & 0.336 & 1 \\
\hline \multirow[t]{3}{*}{ NZSX } & $\mathrm{MM}$ & 1 & 0.848 & 0.272 & 0.032 & 0.02 & 0.016 & 0.252 & 0.764 & 1 \\
\hline & T-to-T & 1 & 0.828 & 0.26 & 0.02 & 0.02 & 0.016 & 0.248 & 0.76 & 1 \\
\hline & Sign T-to-T & 1 & 0.948 & 0.376 & 0.028 & 0.032 & 0.036 & 0.424 & 0.908 & 1 \\
\hline \multirow[t]{3}{*}{ BOLSA } & MM & 1 & 0.588 & 0.156 & 0.024 & 0.024 & 0.016 & 0.128 & 0.52 & 1 \\
\hline & T-to-T & 1 & 0.56 & 0.148 & 0.024 & 0.012 & 0.016 & 0.104 & 0.496 & 1 \\
\hline & Sign T-to-T & 1 & 0.476 & 0.092 & 0.008 & 0.012 & 0.004 & 0.072 & 0.376 & 1 \\
\hline \multicolumn{11}{|c|}{ Panel B: Rank Test, $50 \%$ Increased Variance on Day Zero, and Medium Trading } \\
\hline \multirow[t]{3}{*}{ NZSX } & MM & 1 & 0.692 & 0.24 & 0.044 & 0.016 & 0.02 & 0.28 & 0.74 & 1 \\
\hline & T-to-T & 1 & 0.496 & 0.128 & 0.012 & 0 & 0 & 0.116 & 0.552 & 1 \\
\hline & Sign T-to-T & 1 & 0.944 & 0.516 & 0.088 & 0.056 & 0.056 & 0.556 & 0.964 & 1 \\
\hline \multirow[t]{3}{*}{ BOLSA } & $\mathrm{MM}$ & 1 & 0.844 & 0.296 & 0.036 & 0.028 & 0.04 & 0.24 & 0.832 & 1 \\
\hline & T-to-T & 1 & 0.652 & 0.156 & 0.008 & 0.004 & 0.004 & 0.108 & 0.688 & 1 \\
\hline & Sign T-to-T & 1 & 0.896 & 0.404 & 0.068 & 0.044 & 0.032 & 0.352 & 0.924 & 1 \\
\hline \multicolumn{11}{|c|}{ Panel C: Rank Test, 50\% Increased Variance on Day Zero, and Thin Trading } \\
\hline \multirow[t]{3}{*}{ NZSX } & MM & 1 & 0.768 & 0.236 & 0.024 & 0.02 & 0.044 & 0.304 & 0.796 & 1 \\
\hline & T-to- $\mathrm{T}$ & 1 & 0.328 & 0.032 & 0 & 0 & 0.004 & 0.028 & 0.36 & 1 \\
\hline & Sign T-to-T & 1 & 0.948 & 0.58 & 0.124 & 0.052 & 0.068 & 0.492 & 0.92 & 1 \\
\hline \multirow[t]{3}{*}{ BOLSA } & $\mathrm{MM}$ & 1 & 0.996 & 0.76 & 0.068 & 0.024 & 0.056 & 0.78 & 1 & 1 \\
\hline & T-to-T & 1 & 0.884 & 0.272 & 0.008 & 0.004 & 0.004 & 0.288 & 0.916 & 1 \\
\hline & Sign T-to-T & 1 & 1 & 0.832 & 0.112 & 0.036 & 0.124 & 0.916 & 1 & 1 \\
\hline \multicolumn{11}{|c|}{$\begin{array}{l}\text { This table furnishes, in the presence of a } 50 \text { percent increase in variance on day zero, the rejection rates of the null } \\
\text { hypothesis of no abnormal performance present, when } 250 \text { portfolios of } 50 \text { stocks are employed, and the estimation } \\
\text { period is } 139 \text { days in length and the significance test is the rank test. No medium or thin results are reported for the } \\
\text { NYSE as these could not be generated without massive re-usage of the very few NYSE stocks that met the selection } \\
\text { criteria. }\end{array}$} \\
\hline
\end{tabular}

In Table 9, the first item of note is that the rejection rates of the null hypothesis in the presence of zero induced abnormal performance are either below or close to the 5 percent benchmark for all models, all trading bands, and in all three markets. The second characteristic is that all models reliably detect \pm 5 percent abnormal performance. In addition, the signed trade-to-trade model linked with the rank test is 
able to detect \pm 1 percent abnormal performance on the BOLSA in the thinly-traded band and on the NZSX in all three bands with greater than 90 percent accuracy.

Table 10: Null Hypothesis Rejection with the Rank Test and $100 \%$ added Variance on Day Zero

\begin{tabular}{|c|c|c|c|c|c|c|c|c|c|c|}
\hline \multirow[b]{2}{*}{ Exchange } & \multirow[b]{2}{*}{ Model } & \multicolumn{9}{|c|}{ Percentage of abnormal performance added } \\
\hline & & $-5 \%$ & $-1 \%$ & $-0.50 \%$ & $-0.10 \%$ & 0 & $0.10 \%$ & $0.50 \%$ & $1 \%$ & $5 \%$ \\
\hline \multicolumn{11}{|c|}{ Panel A: Rank Test, $\mathbf{1 0 0 \%}$ Increased Variance on Day Zero, and Thick Trading } \\
\hline \multirow[t]{3}{*}{ NYSE } & MM & 1 & 0.416 & 0.096 & 0.016 & 0.024 & 0.008 & 0.084 & 0.352 & 1 \\
\hline & T-to-T & 1 & 0.412 & 0.096 & 0.016 & 0.028 & 0.008 & 0.084 & 0.356 & 1 \\
\hline & Sign T-to-T & 0.992 & 0.148 & 0.04 & 0.008 & 0.004 & 0.004 & 0.016 & 0.1 & 0.992 \\
\hline \multirow[t]{3}{*}{ NZSX } & $\mathrm{MM}$ & 1 & 0.456 & 0.1 & 0.024 & 0.036 & 0.02 & 0.128 & 0.46 & 1 \\
\hline & T-to-T & 1 & 0.448 & 0.1 & 0.02 & 0.028 & 0.02 & 0.12 & 0.44 & 1 \\
\hline & Sign T-to-T & 1 & 0.472 & 0.168 & 0.024 & 0.032 & 0.024 & 0.116 & 0.492 & 1 \\
\hline \multirow[t]{3}{*}{ BOLSA } & $\mathrm{MM}$ & 1 & 0.268 & 0.108 & 0.02 & 0.016 & 0.016 & 0.056 & 0.312 & 1 \\
\hline & T-to-T & 1 & 0.26 & 0.1 & 0.02 & 0.012 & 0.016 & 0.048 & 0.288 & 1 \\
\hline & Sign T-to-T & 1 & 0.116 & 0.048 & 0.008 & 0.012 & 0.012 & 0.02 & 0.168 & 1 \\
\hline \multicolumn{11}{|c|}{ Panel B: Rank Test, $100 \%$ Increased Variance on Day Zero, and Medium Trading } \\
\hline \multirow[t]{3}{*}{ NZSX } & MM & 1 & 0.328 & 0.084 & 0.008 & 0.016 & 0.032 & 0.072 & 0.304 & 1 \\
\hline & T-to-T & 1 & 0.124 & 0.04 & 0.004 & 0.004 & 0.008 & 0.024 & 0.128 & 1 \\
\hline & Sign T-to-T & 1 & 0.568 & 0.216 & 0.032 & 0.032 & 0.076 & 0.192 & 0.516 & 1 \\
\hline \multirow[t]{3}{*}{ BOLSA } & $\mathrm{MM}$ & 1 & 0.372 & 0.08 & 0.016 & 0.008 & 0.028 & 0.088 & 0.432 & 1 \\
\hline & T-to-T & 1 & 0.164 & 0.024 & 0.004 & 0 & 0.004 & 0.032 & 0.212 & 1 \\
\hline & Sign T-to-T & 1 & 0.472 & 0.152 & 0.032 & 0.02 & 0.032 & 0.132 & 0.52 & 1 \\
\hline \multicolumn{11}{|c|}{ Panel C: Rank Test, $\mathbf{1 0 0 \%}$ Increased Variance on Day Zero, and Thin Trading } \\
\hline \multirow[t]{3}{*}{ NZSX } & MM & 1 & 0.348 & 0.084 & 0.02 & 0.012 & 0.02 & 0.104 & 0.38 & 1 \\
\hline & T-to-T & 0.988 & 0.064 & 0.008 & 0 & 0 & 0 & 0.012 & 0.08 & 0.988 \\
\hline & Sign T-to-T & 1 & 0.556 & 0.212 & 0.088 & 0.048 & 0.084 & 0.236 & 0.572 & 1 \\
\hline \multirow[t]{3}{*}{ BOLSA } & $\mathrm{MM}$ & 1 & 0.812 & 0.228 & 0.032 & 0.036 & 0.028 & 0.292 & 0.868 & 1 \\
\hline & T-to-T & 1 & 0.38 & 0.032 & 0.004 & 0 & 0.004 & 0.036 & 0.312 & 1 \\
\hline & Sign T-to-T & 1 & 0.912 & 0.368 & 0.056 & 0.04 & 0.064 & 0.444 & 0.916 & 1 \\
\hline \multicolumn{11}{|c|}{$\begin{array}{l}\text { This table furnishes, in the presence of a } 100 \text { percent increase in variance on day zero, the rejection rates of the null } \\
\text { hypothesis of no abnormal performance present, when } 250 \text { portfolios of } 50 \text { stocks are employed, and the estimation } \\
\text { period is } 139 \text { days in length and the significance test is the rank test. No medium or thin results are reported for the } \\
\text { NYSE as these could not be generated without massive re-usage of the very few NYSE stocks that met the selection } \\
\text { criteria. }\end{array}$} \\
\hline
\end{tabular}

However, when the standard deviation of returns in the event window is doubled, the superior performance of the signed trade-to-trade model when linked to the rank test evaporates. In Table 10 the \pm 5 percent induced abnormal performance is detected with more than 95 percent accuracy for all markets and at all trading frequencies. All models also correctly handle the nil induced abnormal return 
case with less than a 5 percent error. In addition, the signed trade-to-trade model linked with the rank test identifies \pm 1 percent abnormal performance with more than 90 percent accuracy with respect to the BOLSA's thinly-traded band. But this is an exceptional case. None of the models are consistently reliable at this level with a doubling of event-window standard deviation. However this degree of increase is much larger than the factor 1.2 to 1.5 mentioned by Boehmer et al as expected in the event period.

\section{Conclusions and limitations}

This paper has found that the signed trade-to-trade model is an improvement on the trade-to-trade model that was proposed by Maynes and Rumsey (1993), which had been shown to be an improvement over the market model with missing trades addressed by the lumped method. The improvement that the signed trade-to-trade model makes, however, is market sensitive in that it performs very well indeed on data from small stock exchanges, but in not such a stellar fashion on NYSE data. This is likely to be a function of trading thinness on the two smaller markets.

But given that the world has a large number of small stock markets, this new model has been shown to be capable of making a valuable contribution. In particular, the signed trade-to-trade more accurately diagnoses the presence of artificially imposed abnormal performance especially when the amount of abnormal performance imposed was half a percent or one tenth of a percent. The model continues to perform more accurately also on stocks that are subject to medium-level or thin trading.

While returning better results than the trade-to-trade model, the new model also outperforms the market model. A perusal of the tables in the previous sections will show that the market model is the weakest of the three in this study. This enables this model to operate as well as, if not better than the 
trade-to-trade model on thickly and medium traded stocks, and with a much greater degree of accuracy with respect to thinly traded stocks.

Further, this paper, while not directly addressing the issue, corroborates what other researchers have found, which is that the rank test is the overall best significance test for use in event studies. The combination of the signed trade-to-trade model and rank test generally reduced the incidence of Type I errors; and where there is thin trading, the combination produces both the lowest Type I and Type II errors. The signed trade-to-trade model also exhibited superior performance when the estimation period was approximately halved from 239 days to 111, and then halved again to 61 days. The model also outperformed the trade-to-trade and market models when the size of portfolios was reduced from 50 stocks to 30 and then 20 . However, below that portfolio size none of the three models performed very well. Then, when an artificial increase in variance is imposed on the event day, this performance deteriorates. But so too, do combinations of the rank test with the other two models. This being said, no model or significance test has yet been devised which robustly deals with this problem.

The paper, in passing, also shed some light on the varying natures of regional stock markets and indices. The NYSE, which tends to be thickly traded in the first instance, yielded quite different results from those generated in New Zealand and Mexico. It became clear fairly quickly that the signed trade-totrade model offered little improvement in the NYSE context, but major improvements consistently in the two smaller markets. It did so with value-weighted indices in both of these places whereas the index employed with respect to NYSE data was an equal-weighted one. Corrado and Truong (2008) found that equal-weighted indices furnished superior results to value-weighted ones. The use of company and value-weighted market index returns both in log form in combination with the signed trade-to-trade model might be a viable alternative. 
It is a limitation of this paper that it is relatively narrow in scope and does not investigate, for instance, the effect of clustering of events or replacing day zero with a multi-day event window. These items may well be candidates to be addressed in a further paper.

\section{Bibliography}

Almekinders, G. J., Eijffinger, S. C. W., 1996. A friction model of daily Bundesbank and Federal Reserve intervention. Journal of Banking \& Finance 20, 1365-1380.

Anderson, W., 2009. Alternative event study methodology for detecting dividend signals in the context of joint dividend and earnings announcements. Accounting \& Finance 49, 247265.

Bartholdy, J., Olson, D., Peare, P., 2007. Conducting Event Studies on a Small Stock Exchange. The European Journal of Finance 13, 227-252.

Bartholdy, J., Riding, A., 1994. Thin trading and the estimation of betas: The efficacy of alternative techniques. Journal of Financial Research 17, 241-254.

Bhagat, S., Romano, R., 2007. Empirical Studies of Corporate Law. In: Polinsky, A. M., Shavell, S. (Eds), Handbook of Law and Economics Vol. 2. Elsevier.

Boehmer, E., Musumeci, J., Poulsen, A. B., 1991. Event-study methodology under conditions of event-induced variance. Journal of Financial Economics 30, 253-272.

Brown, S. J., Warner. J. B., 1980. Measuring security price performance. Journal of Financial Economics 8, 205-258.

Brown, S. J., Warner, J. B., 1985. Using daily stock returns : The case of event studies. Journal of Financial Economics 14, 3-31.

Campbell, C. J., Wasley, C. E., 1993. Measuring security price performance using daily NASDAQ returns. Journal of Financial Economics 33, 73-92.

Corrado, C. J., 1989. A nonparametric test for abnormal security-price performance in event studies. Journal of Financial Economics 23, 385-395.

Corrado, C. J., 2009., Event Studies: A Methodology Review. Available at SSRN: http://ssrn.com/abstract=1441581.

Corrado, C. J., Truong, C., 2008. Conducting event studies with Asia-Pacific security market data. Pacific-Basin Finance Journal 16, 493-521. 
Corrado, C. J., Zivney, T. L., 1992. The Specification and Power of the Sign Test in Event Study Hypothesis Tests Using Daily Stock Returns. The Journal of Financial and Quantitative Analysis 27, 465-478.

Cowan, A. R., 1992. Nonparametric Event Study Tests. Review of Quantitative Finance and Accounting 2, 343-358.

Cowan, A. R., Sergeant, A. M., 1996. Trading frequency and event study test specification. Journal of Banking \& Finance 20, 1731-1757.

Dagenais, M. G., 1969. A Threshold Regression Model. Econometrica 37, 193-203.

Dagenais, M. G., 1975. Application of a Threshold Regression Model to Household Purchases of Automobiles. The Review of Economics and Statistics 57, 275-285.

Desarbo, W. S., Rao, V. R., Steckel, J. H., Wind, J., Colombo, R., 1987. A Friction Model for Describing and Forecasting Price Changes. Marketing Science 6, 299-319.

Dimson, E., Marsh, P. R., 1983. The Stability of UK Risk Measures and the Problem of Thin Trading. The Journal of Finance 38, 753-783.

Dimson, E., 1979. Risk measurement when shares are subject to infrequent trading. Journal of Financial Economics 7, 197-226.

Fisher, L., 1966. Some New Stock-Market Indexes. The Journal of Business 39, 191-225.

Forbes, S. M., Mayne, L. S. 1989. A friction model of the prime. Journal of Banking \& Finance 13, 127-135.

Fowler, D. J., Rorke, C. H., Jog V. M., 1989. A Bias-Correcting Procedure for Beta Estimation in the Presence of Thin Trading. Journal of Financial Research 12, 23-32.

Franks, J. R., Broyles, J. E., Hecht, M. J., 1977. An Industry Study of the Profitability of Mergers in the United Kingdom. The Journal of Finance 32:1513-1525.

Hashimoto, Y., Takatoshi, I., 2004. High-Frequency Contagion between the Exchange Rates and Stock Prices. NBER Working Paper Series, No. 10448.

Jokung, O., Meyfredi, J-C., 2003. Improving the Market Model: The 4-State Model Alternative. EDHEC Risk and Asset Management Research Center, Lille Cedex, France

Kallunki, J.-P., 1997. Handling missing prices in a thinly traded stock market: implications for the specification of event study methods. European Journal of Operational Research 103, 186-197. 
Kothari, S.P., Warner, J. B., 2007. Econometrics of Event Studies. In: Eckbo, B. E. (Ed), Handbook of Corporate Finance, Vol. 1. North-Holland: Oxford

Lesmond, D. A., Ogden, J. P., Trzcinka, C. A., 1999. A New Estimate of Transaction Costs. The Review of Financial Studies 12, 1113-1141.

MacKinlay, A. C., 1997. Event Studies in Economics and Finance. Journal of Economic Literature 35, 13-39.

Marsh, P., 1979. Equity Rights Issues and the Efficiency of the UK Stock Market. The Journal of Finance 34, 839-862.

Maynes, E., Rumsey, J., 1993. Conducting event studies with thinly traded stocks. Journal of Banking \& Finance 17, 145-157.

Norsworthy, J., Gorener, R., Morgan, I., Li, D., 2004. Expected Utility, Prospect Theory and Asset Pricing. Proceedings of Canadian Economics Association 38th Annual Meeting. Toronto.

Patell, J. M., 1976. Corporate Forecasts of Earnings Per Share and Stock Price Behavior: Empirical Test. Journal of Accounting Research 14, 246-276.

Rosett, R. N., 1959. A Statistical Model of Friction in Economics. Econometrica 27, 263-267.

Sanders, R. W., Robins, R. P., 1991. Discriminating between wealth and information effects in event studies in accounting and finance research. Review of Quantitative Finance and Accounting 1, 307-329.

Scholes, M., Williams, J., 1977. Estimating betas from nonsynchronous data. Journal of Financial Economics 5, 309-327.

Schwert, G. W., 1977. Stock exchange seats as capital assets. Journal of Financial Economics 4, 51-78.

Zmijewski, M. E., 1984. Methodological Issues Related to the Estimation of Financial Distress Prediction Models. Journal of Accounting Research 22, 59-82. 